\title{
S-B-2. The Location of Sodium in Normal and Edematous Brain Tissue
}

\author{
Eiichi Tani, Hajime Handa, Toshio Ametani and Sadao Kawamura \\ Department of Neurosurgery, Kyoto University Medical School
}

The sodium space in the brain tissue is about 35 per cent, assuming that the electrolyte composition of the extracellular fluid and the plasma is the same. On the other hand, the sodium space is about 24 per cent on the basis of assumption of the same electrolyte composition between the cerebrospinal and the extracellular fluid. The evaluation of the extracellular space by means of electron micrographs and sulphate space as well as impedance indicates about 5 per cent.

The location of sodium has been shown in electron micrography by use of potassium pyroantimonate in the fixatives by Komnick, Kaye, Hartmann, and Zadunaisky. Komnick's or Hartmann's method is most sensitive in test tube, but results in a poor preservation of the brain tissue. In addition, the potassium pyroantimonate easily forms a precipitation of antimonic acid below $\mathrm{pH} 7$. Consequently, the use of acetic or hydrochloric acid in Komnick's or Hartmann's method has possibility to form the antimonic acid, depending upon the method of mixture. The presence of buffer solution in Kaye's or Zadunaisky's method prevents from formation of antimonic acid and makes the brain tissue adequately preserved. Precipitation particles of antimonic acid and sodium pyroantimonate are shown in electron micrographs.

Precipitation particles by Komnick's method was evident throughout the cellular compartments of the brain tissue, except in the capillary basement membrane and the oligodendroglia. Their distribution is rather dense in the cerebral cellular compartments than the capillary lumen. However, they are located compactly in most of astrocytes. There is no definite evidence of their intimate correlation with a certain organella, although they are often located densely in the endoplasmic reticulum or the nuclear gap. On occasion, particles precipitated by Komnick's method are sparsely distributed in the cellular compartment, and rather similar in distribution seen by Kaye's or Zadunaisky's method. It seems not to be due to the location of examined portion, because of no evidence of dense distribution in the superficial portion of specimens. Precipitation particles visualized by Zadunaisky's method, are evident mostly in the capillary lumen and the astrocyte. They are located in vesicles and cytoplasm. Some of them is similar in size to $\beta$-glycogen particles. Few of them are observed in the nerve fiber. Smaller particles are occasionally deposited in nerve fiber, vesicle of astrocyte, non-synaptic vesicles, and myelin sheath. Careful consideration has to be directed to ion movement during fixation. However, to date, our examination of sodium location suggests that the astrocyte is a high sodium compartment in the brain tissue.

The locaion of sodium is examined in edematous brain tissue produced by 
triethyltin and epidural compression. Komnick's as well as Zadunaisky's methods reveal precipitation products in clear space produced by splitting of the myelin sheath in triethyltin intoxication. The precipitation products are also visible in splitting places of the myelin sheath. In addition, smaller precipitations are visualized mainly on the major dense lines of the myelin sheath. Increase in precipitation products is not verified in the cortex. This observation quite agrees with the biochemical evidence. Similar distribution of precipitation products is evident in splitting space of the myelin sheath and on the major dense lines of the myelin in the swollen tissue produced by epidural compression. Precipitation products are occasionally seen in the distended extracellular space. Glycogen granules are increased in number in astrocytes, but there is no definite observation of increased precipitation products.

The distribution of acid mucopolysaccharide is determined by use of ruthenium red. The reaction products filled intercellular gaps and synaptic clefts completely and accumulated in the capillary basement membranes and the myelin sheath. The filling of synaptic clefts is most compact. Occasionally, the reaction products are evident in the membrane of endoplasmic reticulum and the nuclear membrane as well as in the cytoplasm of astrocyte. The intercellular substance, presumably acid mucopolysaccharide, might facilitate the transport of specific ions and has a considerable histophysiological significance.

\title{
S-B-3. Biochemical Study of Brain Edema
}

\author{
Yoshifumi Furusawa, Kenichi Tanimura, Shozo Nakazawa \\ and Yuichi KomaI \\ Department of Neurosurgery, Brain Research Institute, Niigata University
}

Experimental brain edemas were produced in cats and rats by means of intraperitoneal injection of triethyltin sulfate, epidural compression, cold injury and ultrasonic irradiation. In white matter, increase in water, sodium and chloride conents and decrease in potassium were the common phenomena. In gray matter, however, such changes were minimum or negligible. Soluble protein content in both gray and white matters was elevated in all experimental groups except for in case of triethyltin intoxication where no change was demonstrated. By polyacrylamide gel elecrophoresis and agar immunoelectrophoresis examination, the main component of the increased protein in edematous brains was proved to be the albumin of serum origin.

From the above mentioned changes in water, electrocyte and soluble protein contents, it can be deduced that some lesions in active transport mechanisms located in the membranes of cerebral tissues have developed. In estimating the high 